\title{
CLINICAL AND LABORATORY PROFILE OF BRAZILIAN PATIENTS WITH PRIMARY ANTIPHOSPHOLIPID SYNDROME (PAPS): COMPARISON WITH PATIENTS FROM OTHER PARTS OF THE WORLD USING DATA FROM THE ANTIPHOSPHOLIPID SYNDROME ALLIANCE FOR CLINICAL TRIALS AND INTERNATIONAL NETWORKING (APS ACTION)
}

Erivelton de Azevedo Lopes ${ }^{1, \star}$, Gustavo Guimarães Moreira Balbi ${ }^{1,2}$, Michelle Remião Ugolini Lopes ${ }^{1}$, Danieli Castro Oliveira de Andrade ${ }^{1}$ 1.Universidade de São Paulo, São Paulo (SP), Brazil. 2.Universidade Federal de Juiz de Fora, Juiz de Fora (MG), Brazil.

*Corresponding author: erivelton_lopes@yahoo.com.br

\section{BACKGROUND}

The study of the profile of a rare disease like primary antiphospholipid syndrome (PAPS) in a mixed population is important, because it can provide new concepts for understanding additional information in autoimmune diseases. Based on that we evaluated the profile of patients with PAPS in Brazil and compared them with patients from other countries form Antiphospholipid Syndrome Alliance for Clinical Trials and International Networking (APS ACTION) database.

\section{MATERIALS AND METHODS}

We classified patients into two groups: (1) patients from Brazil; and (2) patients from other countries. The two groups were compared regarding demographic profile, clinical criteria, frequency of arterial and venous thrombosis, non-criterion manifestations, antibody profile, risk factors for thrombosis and the adjusted Global Antiphospholipid Syndrome Score (aGAPSS).

\section{RESULTS}

This retrospective multicenter study included 415 PAPS patients. Brazilian patients were younger (mean age of $47.6 \pm 11.3$ vs. $50.7 \pm 13.3$ ), more frequently female (79.5 vs. $65.1 \%, p=0.01$ ) and non-white (69.3 vs. $23.3 \%, p<0.001)$. We found no statistically significant differences between rates of criteria manifestations (venous and arterial thrombosis - including small vessels and CAPS - and obstetric manifestations). Patients from Brazil presented more frequently with livedo (25 vs. 10.4\%, p<0.001), cognitive dysfunction (20.5 vs. $8 \%, p=0.001$ ) and seizures (15.9 vs. 6.7\%, $p=0.007$ ), and less frequently with thrombocytopenia ( 9.1 vs. $18.3 \%, p=0.037$ ). Brazilian patients had a higher frequency of lupus anticoagulant ( $87.5 \mathrm{vs} .74 .6 \%, p=0.01$ ) and a lower frequency of anticardiolipin (aCL) (46.6 vs. 73.7\%, p < 0.001) and anti-B2-glycoprotein I (aß2GPI) (13.6 vs. 62.7\%, p<0.001). When assessing the sole presence of a single antibody, patients with only aCL (9.1 vs. 7.0\%, p 0.52) and only aß2GPI (0 vs. 4.0\%, p 0.52) were similar in both groups. However, patients positive to LA only were more frequent in the Brazilian group ( $51.1 \mathrm{vs.} 19.6 \%, p<0.001$ ). The rates of double positivity were similar between groups (31.8 vs. 27.8\%, p 0.46), but triple positivity was less frequent in Brazilian patients ( 8 vs. $41.6 \%, p<0.001$ ). Patients from Brazil were more often sedentary ( 80.7 vs. $33.6 \%, p<0.001$ ) and obese (36.4 vs. $22 \%$, $p=0.006$ ). The median aGAPSS was 8 (4-9) for Brazilian patients and 10 (8-13) for patients from other countries, which was statistically significant $(p<0.0001)$.

\section{CONCLUSION}

Primary antiphospholipid syndrome in the Brazilian population occurs more frequently in women of non-white ethnicity and the patients were more sedentary and obese. Livedo, cognitive dysfunction and seizures were more frequent in this group, when compared to other countries. They also had a higher frequency of lupus anticoagulant and lower frequency of anticardiolipin, antiB2Glycoprotein-I and triple positivity. 